\title{
Determining Method for Potential Injury Attitude Based on Three- dimensional Image Analysis
}

\author{
yuehaifeng \\ Dongying Vocational College, shandong, dongying, 257091
}

Keywords: three-dimensional image; potential sports; sports injuries

\begin{abstract}
Potential injured area of athletes by image analysis may produce a frontier technology. In the method for determining the attitude of sports injuries, the potential injury-prone parts such as the wrist, waist and knees and other more hidden location during image capture almost can not guarantee the continuity of image acquisition, resulting in discontinuous movement in image features, which is hard to form reasonable judgment. Three-dimensional positioning method of motion magnitude is proposed on basis of ML, jointing three-dimensional dynamic model of the target, readily obtaining information of radial distance, azimuth and Doppler of athlete according to visual data, establishing the maximum likelihood estimation on moving amplitude in threedimensional target positioning, and achieve judgment of the target. Simulation results show that the potential sports injuries pose based on three-dimensional image analysis can effectively improve judging accuracy of injury attitude, internal division of media space distribution of injury, reducing injury probability in the long course and providing better protection for potential movement.
\end{abstract}

\section{Introduction}

Currently individual sports are in sustained rapid development, community healthy needs is gradually expanding [1], the judgment of potential injury caused by sports attacks is paid more and more attention [2]. Prevention demand of sports injuries as an important potential sports risks also increases [3, 4]. Considering correlation of sports injuries and psychological factors, such as, risk perception, risk responsibility, athletic ability determination, for public health population in perspective of sports psychology [5], determining experienced validity of the above factors on the actual fitness training in sports injuries, and exploring gender differences between them [6,7], provide a theoretical basis for further reducing and preventing sports injuries. At present, research platform for potential sports injury, to a certain extent, achieve basic injury judgments against injuries to provide self-protection and prevention by sports coaches and other professionals $[8,9]$. Tutorial prevention services of sports injuries are only provided for the internal sports professionals. Due to the various sports organizations with various integrated data [10], different prevention ways, it is not accurately to determine the potential injury, resulting in personnel duplication and resources waste. Potential injury prevention of sports is widely used in the field of large-scale sports, as a new concept of prevention so as to form concept in all sports injury environment, by efficient and convenient analysis of injury data information and processing.

\section{Three-dimensional positioning algorithm of motion amplitude estimation based on ML}

Three-dimensional positioning modeling of motion amplitude. Assuming one motion amplitude locates at $(x, y, z)$. The target's initial position is $x_{0}=\left(x_{0}, y_{0}, z_{0}\right)$, with uniformed motion at $v=\left(v_{x}, v_{y}, v_{z}\right)$ speed,while at $k$ time target's location $x_{k}=\left(x_{k}, y_{k}, z_{k}\right)$ is described as:

Where, $T_{0}$ is sampling interval.

$$
\left\{\begin{array}{l}
x_{k}=x_{0}+k T_{0} v_{x} \\
y_{k}=y_{0}+k T_{0} v_{y} \\
z_{k}=z_{0}+k T_{0} v_{z}
\end{array}\right.
$$

Devices can readily access target's radial distance, azimuth and Doppler information based on video data. The radial distance of the target can be determined based on the target's delay; the 
actual distance $R_{k}$ at time $\mathrm{k}$ between the target devices will be described in Equation (2)

$$
R_{k}=\sqrt{\left(x_{k}-x\right)^{2}+\left(y_{k}-y\right)^{2}+\left(z_{k}-z\right)^{2}}
$$

Relationship between actual azimuth and target position $x_{k}$ at time $k$ can be expressed in equation (3):

$$
\theta_{k}=\arctan \left(\frac{y_{k}-y}{x_{k}-x}\right)
$$

When the collection device and the target have the relative motion, the device receives an additional Doppler shift component, relationship between the target position $x_{k}$ at time $k$ can be described as:

$$
f_{k}=-\frac{2}{\lambda}\left(x_{k}, y_{k}, z_{k}\right)\left[\begin{array}{c}
x_{k}-x \\
y_{k}-y \\
z_{k}-z
\end{array}\right] / R_{k}
$$

where, $\lambda$ is wave length of collection device.

In practical applications it is incapable to obtain real information of moving targets, range of motion measurements generally have a random error. Therefore, at the time $k$ the measurement results of the athletes can be described as:

$$
\left\{\begin{array}{l}
\tilde{R}_{k}=R_{k}+\Delta R_{k} \\
\tilde{\theta}_{k}=\theta_{k}+\Delta \theta_{k} \\
\tilde{f}_{k}=f_{k}+\Delta f_{k}
\end{array}\right.
$$

where, $\tilde{R}_{k}$ means athletes' distance, $\tilde{\theta}_{k}$ means azimuth, $\tilde{f}_{k}$ means Doppler information at time $k$; $\Delta R_{k}, \Delta \theta_{k}$ and $\Delta f_{k}$ respectively means reference error. Assuming errors are in Gaussian distribution, its values are depending on signal-noise ratio.

For convenience, testing data at time $M+1$ is presented as:

$$
\left\{\begin{array}{l}
\tilde{R}=R_{M}+\Delta R_{M} \\
\tilde{\theta}=\theta_{M}+\Delta \theta_{M} \\
\tilde{f}=f_{M}+\Delta f_{M}
\end{array}\right.
$$

where, $\Delta R_{M}, \Delta \theta_{M}$ and $\Delta f_{M}$ respectively means error set of $(M+1) \times 1$ dimensional distance, azimuth and Doppler information. In Gaussian distribution, covariance matrix $Q_{R_{M}}, Q_{\theta_{M}}$ and $Q_{f_{M}}$ are described as follows

$$
\left\{\begin{array}{l}
Q_{R_{M}}=\operatorname{diag}\left\{\sigma_{R_{0}}^{2}, \sigma_{R_{1}}^{2}, \cdots, \sigma_{R_{M}}^{2}\right\} \\
Q_{\theta_{M}}=\operatorname{diag}\left\{\sigma_{\theta_{0}}^{2}, \sigma_{\theta_{1}}^{2}, \cdots, \sigma_{\theta_{M}}^{2}\right\} \\
Q_{f_{M}}=\operatorname{diag}\left\{\sigma_{f_{0}}^{2}, \sigma_{f_{1}}^{2}, \cdots, \sigma_{f_{M}}^{2}\right\}
\end{array}\right.
$$

Based on the above measurement data at time $M+1$, it is optimized the identification of the target in three-dimensional position.

Joint optimization of three-dimensional positioning of the target recognition.Converting Equation (1) (7) to re-described as a function of the moving target state $\varphi$

$$
\left\{\begin{array}{l}
R_{k}(\varphi)=\sqrt{\begin{array}{l}
\left(x_{0}+k T_{0} v_{x}-x\right)^{2}+\left(y_{0}+k T_{0} v_{y}-y\right)^{2} \\
+\left(z_{0}+k T_{0} v_{z}-z\right)^{2}
\end{array}} \\
\theta_{k}(\varphi)=\arctan \left(\frac{y_{0}+k T_{0} v_{y}-y}{x_{0}+k T_{0} v_{x}-x}\right) \\
f_{k}(\varphi)=-\frac{2}{\lambda} \cdot\left(v_{x}, v_{y}, v_{z}\right) \cdot\left[\begin{array}{l}
x_{0}+k T_{0} v_{x}-x \\
y_{0}+k T_{0} v_{y}-y \\
z_{0}+k T_{0} v_{z}-z
\end{array}\right] / R_{k}(\varphi)
\end{array}\right.
$$


where, $k=0,1, \cdots, M$ is time subscript.To analyze in the perspective of spatial analytic geometry, when $M>2$,Equation ( 8 ) is solvability, one solution is actual location of target.ML (maximum likelihood estimation) of the cost function can be described as

$$
\underset{\varphi}{\arg \min }\left\{\left(\tilde{\eta}_{M}-d(\varphi)\right)^{T} W^{-1}\left(\tilde{\eta}_{M}-d(\varphi)\right)+\varepsilon g(\varphi)\right\}
$$

where, $\varepsilon$ expresses as penalty factor; $g(\varphi)$ means obstacle function.

$$
g(\varphi)=\frac{1}{(\varphi(3)-z)}
$$

$\tilde{\eta_{M}}$ is set measured before time $M+1 ; d(\varphi)$ is represented as:

$$
d(\varphi)=\left[\cdots, R_{i}(\varphi), \cdots, \theta_{i}(\varphi), \cdots, f_{i}(\varphi), \cdots\right]^{T}
$$

$W$ is the observation noise covariance matrix. Assuming that the matrix $W$ is a unit matrix, and all measurement errors are known, a least squares estimation is obtained in target state, described by the following equation:

$$
W=\operatorname{diag}\left\{\tilde{Q}_{R_{M}}, \tilde{Q}_{\theta_{M}}, \tilde{Q}_{f_{M}}\right\}
$$

where, $\tilde{Q}_{R_{M}}, \tilde{Q}_{\theta_{M}}$ and $\tilde{Q}_{f_{M}}$ respectively means covariance matrix of identified distantce, location and Doppler error.

Solution process of formula (12) is considered as a non-linear least-squares optimization process, firstly at $\varphi=\varphi_{0}$ location, $d(\varphi)$ will be converted to a linear expression:

$$
d(\varphi)=d\left(\varphi_{0}\right)+D\left(\varphi_{0}\right)\left(\varphi-\varphi_{0}\right)
$$

where, $\varphi_{0}$ is initial solution of target state; $D\left(\varphi_{0}\right)$ is $3(M+1) \times 6$ Jacobian matrix:

$$
D\left(\varphi_{0}\right)=\left[P_{R}^{T}, P_{\theta}^{T}, P_{f}^{T}\right]^{T}
$$

Then,ML estimates can be calculated to obtain the minimum value of the moving target state

$$
\begin{aligned}
& \varphi=\varphi_{0}+\left(D\left(\varphi_{0}\right)^{T} W^{-1} D\left(\varphi_{0}\right)+\varepsilon \frac{\partial^{2} g(\varphi)}{\partial \varphi^{2}}\right)^{-1} \\
& \left(D\left(\varphi_{0}\right)^{T} W^{-1}\left(\eta_{M}-d\left(\varphi_{0}\right)\right)+\varepsilon \frac{\partial g(\varphi)}{\partial \varphi}\right)
\end{aligned}
$$

The newly acquired $\varphi$ as an initial value $\varphi_{0}$ at the next time to continue the iteration with the penalty factor $\varepsilon=\varepsilon / 2$, the above process is repeated until the difference between two successive recognition results of optimization does not exceed a predetermined threshold value $\varphi-\varphi_{0}<\gamma$. At the moment that $\varphi$ is acquired as ML estimation of the target state, while the first three elements of $\varphi$ obtain the spatial location of the target.

\section{Simulation experiment}

The simulation-based three-dimensional image acquisition method is injected into motion environment system to determine potential sports injury. Taking one sports staff as example, sports environment and three-dimensional image acquisition parameters were set. Potential sports injuries pose are determined in the three-dimensional image analysis based on simulation experiments.

In the sports environment for multiple samples collected on the traditional methods and new methods of injury analysis, it is shown in Figure 1: 


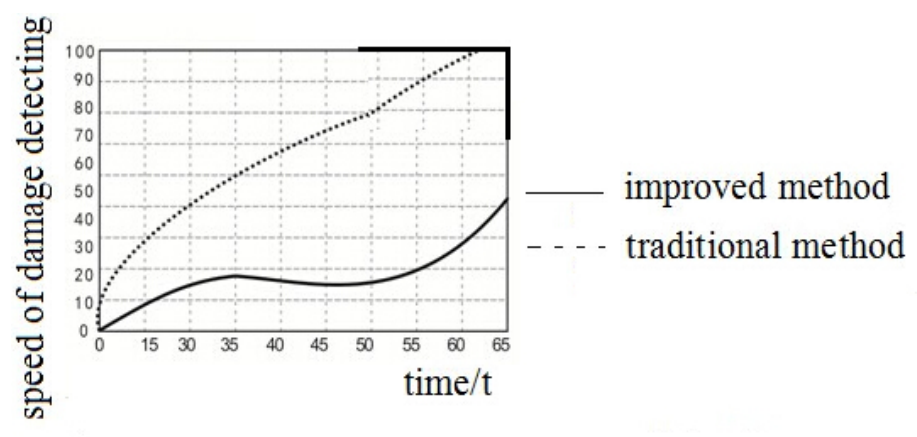

Figure1 injury determining speed between traditional and improved method

In Figure 1, $\mathrm{x}$ axis represents time, y-axis represents the speed of sports injuries. With different testing methods, traditional detection is slower and less efficient.

In the motion environment to collect samples to analysis the conventional method and the new method, accuracy for determination of injury are shown in Figure 2:

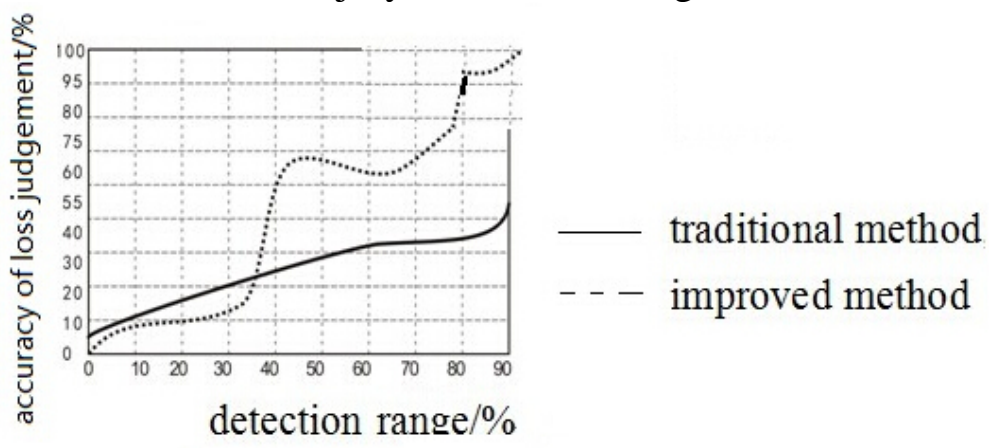

Figure 2 determining accuracy of traditional and improved method

In Figure 2, x-axis means the detection range. y-axis represents the judgment accuracy of sports injuries. In the same detection range, the traditional method has lower accuracy than improved ones.

In the sports environment, analysis of the extent of sports injuries is shown in Figure 3:

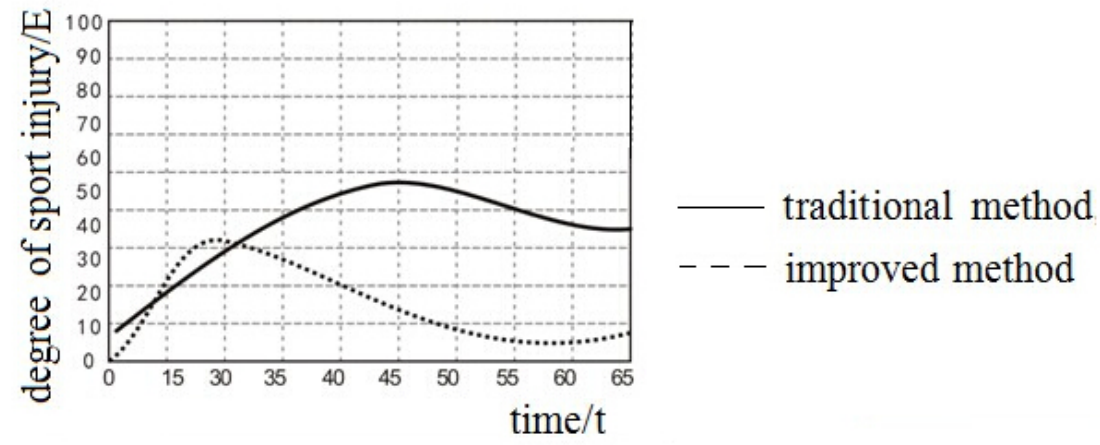

Figure 3 sports injury degree between traditional and improved methods

In Figure $3 \mathrm{x}$ axis represents time, $y$-axis represents the degree of sports injuries. With the same simulation time, the traditional method of sports suffers greater injury.

Simulation results show that the potential injury gesture analysis in three-dimensional image simulation, improves incomprehensive limits of the original sports injury data services, which is carried out only under the guidance of professional staff training; improve continuity of injuryprone part, which directly manifest injury prevention features and reasonable divide internal media space of sports joints. It has advantages of reducing the risk of potential sports injuries, improving speed and accuracy of potential injury judgment, greatly reducing the injury chances of sports staff, improving the efficiency of injury identification, avoiding the waste of resources.

\section{Conclusions}

Through analysis of determination method's shortage of sports potential injuries gesture at this stage, determining simulation is proposed on potential injury gesture on basis of three-dimensional 
image analysis. It is employed to solve inaccurate or not timely judgment of potential injuries for sports staff and satisfy to capture three-dimensional gesture image at high-speed. It is effectively to improve the prevention of sports injuries and provide personnel safety and health protection.

\section{References}

[1] Zhu Hongping,Yu Jing,Zhang Junbing. A summaryreview and advantages of vibration-based damage identification methods in structural health monitoring[J].Engineering Mechanics,2011,28(2):1-11(in Chinese)

[2] Jiang Shaofei, Wu Zhaoqi. Structural health monitoring and intelligent information processing technology and application [M]. Beijing: Chinese Architecture Industry Press, 2011

[3] Reynders E, Teughels A,De Roeck G. Finite element model updating and structural damage identification using OMAX data [J]. Mechanical Systems and Signal Processing, 2010, 24(5)1306-1323

[4] Willems R.M.,Hagoort P.Hand Preference influences neural correlates of action observation[J].Brain Res,2009,1269:90-104

[5] Zhao Ling. the injury investigation and research in sports and fitness activities [J]. Journal of Jingmen Vocational College, 2009, 24(2):85-88.

[6] Casasanto D.,JasminK.Good and bad in the hands of politicians:Spontaneous gestures during positive and negative speech[J].Plos one,2010,5(7):1-5

[7] Guo Jianpeng, Feng Rui. Situation of sports injury investigation of the students majoring in Aerobics of Sports Institute in Shanxi Normal University [J]. Fighting - Sports Forum [Human Sports Science], 2011, 1

[8] M Paladini,A D Bue and M Stosic. Factorization for non-rigid articulated structure using metric projections[C].In2009:2898-2905

[9] Zhang Hongxue. Mechanical factors analysis of basketball sports injury [J]. Journal of Henan Normal University, 2010 (5)

[10] Xiao Liqing, Shao Xiaogen, Wang Linlin. The analysis and design of finite element simulation model for electrical resistance tomography [J]. Chinese Journal of scientific instrument, 2008 (29)2

[11] S.M. Fahimifard, M. Salarpour, M. Sabouhi, S. Shirzady. Application of ANFIS to agricultural economic variables forecasting case study: Poultry retail price [J]. Journal of Artificial Intelligence, 2009, 2 (2), 65-72.

[12] A.D. Niros, G.E. Tsekouras. A novel training algorithm for RBF neural network using a hybrid fuzzy clustering approach [J].Fuzzy Sets and Systems, 2012, 193, 62-84.

[13] Y. Zuo, E. Kita, Stock price forecast using Bayesian network [J]. Expert Systems with Applications, 2012, 39(8), 6729-6737. 